\title{
Initial defence responses in sapwood of Eucalyptus nitens (Maiden) following wounding and fungal inoculation
}

\author{
K. M. BARRY ${ }^{1,2 *}$, R. B. PEARCE ${ }^{4} \dagger, S \cdot D \cdot$ EVANS $^{5}, L \cdot D \cdot H_{L} L^{5}$ and C. M. MOHAMMED ${ }^{1,2,3}$ \\ ${ }^{1}$ CRC for Sustainable Production Forestry, GPO Box 252-12, Hobart, Tasmania, Australia 7001, ${ }^{2}$ School of Agricultural Science, \\ University of Tasmania, GPO Box 252-54, Hobart, Tasmania, Australia 7001, ${ }^{3}$ CSIRO Forestry and Forest Products, GPO Box \\ 252-12, Hobart, Tasmania, Australia 7001, ${ }^{4}$ School of Biological Sciences, University of Birmingham, Birmingham, B15 2TT, \\ U.K. and ${ }^{5}$ Herchel Smith Laboratory for Medicinal Chemistry, University of Cambridge, University Forvie Site, Robinson Way, \\ Cambridge, CB2 2PZ, U.K.
}

(Accepted for publication November 2000 and published electronically 26 January 2001)

\begin{abstract}
Xylem defence responses occurring in pot-grown Eucalyptus nitens (Maiden) saplings were analysed within the first few weeks following stem wounding and inoculation with the non-aggressive decay fungus Ganoderma adspersum (Schulz). The fungus colonized the dead xylem cells up to $5 \mathrm{~mm}$ above and below the wound after 30 days. Evidence of xylem cell death and discolouration was detectable within $12 \mathrm{~h}$ and vessel tyloses were present after 3 days. Concentration of tetra-galloylglucose increased by $3 \cdot 5$-fold within $24 \mathrm{~h}$, while the trend for total phenols was a slow increase reaching a maximum after 21 days. Suberin was not detected. Early accumulation of water content has been proposed as a defence component of hostpathogen interactions in woody angiosperm xylem, but this was not obvious for E. nitens. Nuclear magnetic resonance imaging (NMR imaging or MRI) was used to measure small-scale distributions in moisture content around the inoculated stem wounds in intact E. nitens saplings. Some moisture accumulation was detectable at lesion margins of wounds analysed after 3 weeks. However, this was not detectable by gravimetric measurements. This increase in moisture content may be a consequence of wound physiology rather than an active defence response. $\quad$ (C) 2001 Academic Press
\end{abstract}

Keywords: Eucalyptus nitens; tree; Ganoderma adspersum; antimicrobial defence; reaction zone; phenols; xylem water; nuclear magnetic resonance imaging.

\section{INTRODUCTION}

The response of living sapwood to wounding and subsequent fungal infection is complex. The divide between wound- and infection-related responses remains unclear [23] and infection usually encompasses a range of microorganisms in successional colonization $[4,33,34]$. The secondary xylem represents a hostile environment (e.g. lignified cell walls and low oxygen access) and when this is compromised by wounding active responses are elicited. The active processes occurring shortly after wounding (i.e. the first hours or days) may determine the ability of the tree to restrict the long-term spread of decay resulting from infection $[1,14,23-25]$. Increased oxygen access following wounding has been linked to the production of antimicrobial phenolics $[8,29]$ while changes in vessel osmotic potential can lead to the formation of tyloses [20]. The formation of tyloses and

* To whom all correspondence should be addressed. E-mail: karen.barry@ffp.csiro.au

$\uparrow$ Deceased April 1999. phenolics as defence responses appear ubiquitous among woody plants $[23,37]$. Moisture accumulation at lesion boundaries has been observed in some angiosperms and may present an active sapwood defence response [26, 27]. Suberization of xylem cells may also occur as a defence response, possibly representing a "water-proofing" barrier [22]. Zones of defence responses occurring at lesion boundaries have been termed reaction zones [30-32], column boundary layers $\{\mathrm{CBL}[35]\}$ or CBLreaction zones [23].

Pearce [23] proposed a model of factors contributing to host-pathogen interactions in living angiosperm sapwood, based mainly on studies of Acer spp. This included drying of xylem tissues resulting from wounding, followed by accumulation of phytoalexins within $24 \mathrm{~h}$ and accumulation of water at the lesion margins within 4-7 days. Later stages involved the formation of a more permanent boundary including insoluble polyphenolics and/or suberin. The current study aims to determine whether these key events are typical of other angiosperms, such as the evergreen Eucalypts. 
Recent studies of distinct Eucalyptus nitens reaction zones (formed in response to decay associated with naturallyinfected pruning wounds) found a number of differences in various properties compared to the reaction zones reported from other angiosperms [3]. For example, potassium levels, $\mathrm{pH}$ and water content were lower in the E. nitens reaction zone than healthy sapwood. These traits are typically increased in reaction zone tissue of other angiosperms. These differences may be reflected in the initial stages of host-pathogen interaction. Attributes of the reaction zone that were common to other angiosperms included significantly higher phenol levels than the healthy sapwood and abundant tyloses [2] Concurrent studies have recently revealed the nature of these phenols, which are dominated by hydrolysable tannins [2].

Destructive methods of determining wood moisture content (i.e. gravimetric methods) are used routinely $[8,26,29]$. However, this method is limited to relatively large wood blocks where immediate water loss upon preparation is reduced in significance. To sensitively study the changes in xylem moisture content following wounding and infection, a non-destructive method is essential. The use of nuclear magnetic resonance imaging (NMR imaging or MRI) for the study of intact saplings has provided a very sensitive means of studying moisture levels. The potential to use NMR to image water in whole plants was investigated in the 1980s [e.g. 6, 7, 36] and has been reviewed [16]. Pearce and co-workers [26, 27] have shown that NMR imaging can be used to study woody tissues with decay lesions using both stem segments and intact Acer pseudoplatanus trees.

E. nitens is an important hardwood plantation species in Tasmania. This paper is the first description of initial alterations and defence responses associated with stem wounding and infection in Eucalyptus. Pot-grown E. nitens saplings were notch-wounded and challenged with Ganoderma adspersum. While the NMR imaging studies reported are relatively preliminary, results show that a non-invasive approach to monitor sensitive changes in moisture content are informative.

\section{MATERIALS AND METHODS}

\section{Plant and fungal material}

Pot-grown E. nitens saplings between 10 and 11 months of age were used. E. nitens seed (Toorongo provenance) was provided by Forestry Tasmania (Perth, Tasmania). Seedlings were raised in a glasshouse and transferred to $17 \mathrm{~cm}$ diameter pots filled with commercial potting mix, subject to frequent liquid fertilizer and daily watering. Pot-grown trees were maintained in a poly-tunnel at the University of Birmingham, U.K. Experiments began in August 1998 (summer) when the trees were over $1 \mathrm{~m}$ in height with abundant side branches. Secondary xylem in the saplings was well developed by this stage and stems were up to $2 \mathrm{~cm}$ in diameter.

Cultures of G. adspersum (Schulz.) were maintained on $3 \%$ malt agar in the dark at $25^{\circ} \mathrm{C}$.

\section{Experimental design}

Different sets of trees were used to facilitate a range of studies of initial defence responses. Fifteen trees were used for microscopic examination and phenol analysis at a range of times following wounding and inoculation. For moisture content studies eight trees (plus one for preliminary examination) were utilized also at a range of times since wounding and inoculation. An additional tree was utilized for studies of potassium concentration at one time point after wounding and inoculation.

\section{Wounding and inoculation}

Pot-grown saplings were notch-wounded with a small chisel, creating a wound $6 \mathrm{~mm}$ wide, $18 \mathrm{~mm}$ long and approx. 4-5 mm deep. Rectangular pieces of $G$. adspersum culture ( $3-5$ weeks old) were adpressed to the wound and sealed with parafilm.

\section{Anatomical, histochemical and phenol analyses}

Fourteen 10 month old E. nitens were subject to two inoculated wounds each. The first wound was approx. $15 \mathrm{~cm}$ above ground level on the stem and the second wound was approx. $30 \mathrm{~cm}$ above ground level on the opposite side of the stem. One un-wounded tree was destructively analysed as a control, using stem material from within the first $40 \mathrm{~cm}$ of stem. Destructive sampling of two trees (comprising four wounds) took place at seven time intervals (12 and 24 h, 3, 7, 14, 21 and 30 days). For each plant at each time interval, the stem was dissected and a segment (approx. $10 \mathrm{~cm}$ ) containing the wound was cut through axially. The extent of discolouration and decay associated with the inoculated wound was noted immediately.

Wood blocks for sectioning were removed from an axially-cut stem half of one wound per tree. One piece was prepared from the middle of the wound to $1 \mathrm{~cm}$ above, and a second piece from the middle to $1 \mathrm{~cm}$ below the wound. Transverse and radial longitudinal sections (20-60 $\mu \mathrm{m})$ were cut using a sledge microtome. Wood blocks were sectioned without fixation or embedding and examined on the day of harvest. For general wood anatomy, $1 \%$ toluidine blue in a $0.2 \mathrm{M}$ phosphate buffer ( $\mathrm{pH}$ 6.5) was used. Fast blue RR salt was freshly prepared (FBRRS, $0 \cdot 5 \%$ aqueous, Sigma Chemical Co.) and utilized to stain phenolics, resulting in a red 
colouration. To detect suberin, sections were stained with phloroglucinol-HCl and observed with fluorescence microscopy $[28]$. To visualize viable parenchyma cells, sections were placed in a buffered solution of nitro-blue tetrazolium (1 mg NBT in $1 \mathrm{ml}$ phosphate buffer, $\mathrm{pH} 7 \cdot 1)$ added to equal parts of NADH $(7 \mathrm{mg}$ in $1 \mathrm{ml}$ buffer) for $3 \mathrm{~h}$ in the dark [28]. NAD diaphorase activity is indicated by a purple colour. This was also useful to detect fungal hyphae. Fungal isolation from challenged sapwood was carried out by excising wood chips from xylem of decayed appearance at the 21 and 30 day stage (where enough decay was visually present). Wood chips were incubated at $25^{\circ} \mathrm{C}$ in the dark on $3 \%$ malt agar. Isolated fungi were subsequently sub-cultured and G. adspersum was identified based on morphological characteristics.

The other half of each wound was utilized immediately for chemical extraction. Reaction zone tissue was sampled from within $2 \mathrm{~cm}$ above and below the wound, adjacent to apparently infected or decayed tissue. Healthy sapwood was also sampled from a position on the opposite side of the stem, at least $5 \mathrm{~cm}$ below the centre of the wound. Between 20 and $100 \mathrm{mg}(\mathrm{FW})$ of thinly-sliced wood material was obtained with a small chisel and then extracted twice in $500 \mu \mathrm{l}$ of $100 \%$ methanol for $24 \mathrm{~h}$ at $4^{\circ} \mathrm{C}$. Extracts were then pooled to yield a $1 \mathrm{ml}$ extract and particulate matter was removed by centrifugation. Extracts were kept at $-20^{\circ} \mathrm{C}$ in the dark and analysed during the following weeks.

Total phenols were determined by the Folin-Ciocalteu method, as previously described [5]. Absorbance of the solutions was measured at $725 \mathrm{~nm}$ with a spectrophotometer (LKB Biochrom Ultrospec II E). Concentrations of total phenols were calculated with reference to a gallic acid standard curve $\left(2-40 \mu \mathrm{g} \mathrm{ml}^{-1}\right)$ after regression analysis and expressed as gallic acid equivalent $\left(\mathrm{mg} \mathrm{gFW}^{-1}\right)$.

Extracts were also analysed by HPLC. The system consisted of two LDG Constametric III pumps (LCD Ltd, Stone, Staffordshire, U.K.), an LDG Spectromonitor III spectrophotometer and a Spectra-Physics SP4270 Integrator. A C18 Spherisorb ODS analytical column $(4.6 \times 250 \mathrm{~mm})$ was used with a C18 Spherisorb ODS guard column $(4.6 \times 10 \mathrm{~mm})$. The solvent program was a linear gradient from $100 \%$ solvent A ( $\mathrm{MeOH}: \mathrm{H}_{2} \mathrm{O}:$ acetic acid, $5: 92: 3$ ) to $100 \%$ solvent B $(100 \% \mathrm{MeOH})$ over $40 \mathrm{~min}$ with a further $4 \mathrm{~min}$ at $100 \% \mathrm{~B}$ before returning to the initial conditions. Compounds were detected at $260 \mathrm{~nm}$, and external gallic acid standards $(5-10 \mu \mathrm{g})$ were used to obtain semiquantitative data for particular peaks. Peak height was measured, as the chromatographic separation did not allow accurate measurement of peak area.

\section{Moisture content studies (NMR imaging and gravimetric)}

Following preliminary NMR imaging studies, seven trees were notch-wounded and inoculated as described above (approx. $35 \mathrm{~cm}$ from the base of the tree). Stems from different individual trees were imaged at seven stages after wounding (within 1 and $24 \mathrm{~h}$ and 4, 7, 15, 22 and 72 days). An unwounded stem was imaged $35 \mathrm{~cm}$ from the base of the tree as a control. Imaging was done at the Herchel Smith Laboratory, Addenbroke's Hospital, Cambridge, U.K. All images were acquired at room temperature using a 2.35 Tesla, $31 \mathrm{~cm}$ horizontal bore superconducting magnet connected to a Bruker BMT (Bruker Medzintechnik Biospec II, Karlsruhe, Germany) imaging console. A $15 \mathrm{~cm}$ internal diameter gradient set built "in-house" which produces approx. 0.1 Tm-1 gradient fields was used, together with a cylindrical eight strut birdcage probe (internal diameter $9.4 \mathrm{~cm}$ ) operated in the quadrature mode to transmit and receive the signal. The probe was firmly positioned around the stem using foam inserts, with the wound directly in the centre of the probe.

In outline, the signal generated from NMR imaging studies largely corresponds to the "mobile" protons of water in the sample. Data is first acquired as a "spinecho" in which a number of factors contribute to the image contrast, including the abundance of protons as well as factors relating to the molecular mobility of those protons. That is, the time the protons take to return to equilibrium after a radio-frequency pulse ( $T_{1}$ relaxation) and the time taken to go "out of phase" with the magnetic field $\left(T_{2}\right.$ relaxation) will increase with greater mobility and effect the signal. Data for $T_{1}$ and/or $T_{2}$ relaxation times can be obtained, enabling data relating closely to proton abundance $\left(M_{0}\right.$ data) to be calculated. Preliminary studies revealed that $T_{1}$ relaxation did not greatly affect the signal, therefore $T_{2}$ data was acquired alone.

Transverse and longitudinal pilot images were acquired using a spin-echo imaging sequence with inter-echo time of $20 \mathrm{~ms}$. These pilot images were used to align the desired imaging plane longitudinally through the wound. The quantification of MR parameters was achieved by varying the number of echoes in the multiecho Carr Purcell Meiboom Gill MRI sequence [18]. The $T_{2}$ values were calculated from images with different TE (inter-echo) values [eight images, inter echo time $20 \mathrm{~ms}$, repetition time (TR) $3 \mathrm{~s}$ ] using the following equation;

$$
M_{x y}=M_{0} e^{\left(-1 / T_{2}\right)}+C
$$

where $M_{x y}$ is the magnetization at a particular pixel; $C$ is the estimate of the average noise in the images; $M_{0}$ and $T_{2}$ are the estimates required for starting the iterative 
curve-fitting process based on the Levenberg-Marquardt method [17]. The actual $M_{0}$ value was quantified by back-projection of the $T_{2}$ decay curve to where it intersected the $y$-axis at time zero.

Images had a field of view (FOV) of $7 \mathrm{~cm}$, imaging slice thickness of $2 \mathrm{~mm}, 256$ frequency encoding points in read direction (along the stem) and 128 phase encoding points across the stem. A FOV extension factor of two in the read direction to prevent aliasing gave images 512 by 128 pixels that were processed to give images 256 by 256 pixels with a resolution of $273 \mu \mathrm{m}$ (7 cm divided 256) per pixel. With four averages, the acquisition time was approx. 26 min (128 phase encode steps * 4 averages * TR) per image.

$M_{0}$ and $T_{2}$ values were calculated as the average of a circular area covering about 40 pixels from the $M_{0}$ and $T_{2}$ maps using "Cmrview" software. These circular areas were chosen to correspond to prominent areas of contrast and to match the position of samples taken for gravimetric determinations. The $M_{0}$ and $T_{2}$ values were calculated three times and a standard error was determined for each area. As this standard error was typically a factor of $10^{3}$ lower than the $M_{0}$ average, it has not been presented in the results. Unfortunately, the signal to noise ratio was not sufficient to enable accurate $M_{0}$ values to be determined from two of the trees. To facilitate comparison of tissue moisture content from tree to tree the ratio of reaction zone to the corresponding healthy sapwood on the opposite side of the stem was calculated for $M_{0}$ and gravimetric data.

Trees were dissected as soon as possible after imaging for gravimetric determinations of moisture content. Bark was removed and blocks of wood $\left(0.8 \mathrm{~cm}^{3}\right.$ minimum) were excised with a chisel from positions around the wound and in corresponding positions on the other side of the stem. For the stems with 22 and 72 day old wounds, two smaller blocks were excised from above and below the wound, in an attempt to include only material related to areas of bright contrast evidenced by NMR imaging. For the control tree, four blocks were excised around the centre of the image area. Fresh weight was determined immediately. Samples were then dried at $85^{\circ} \mathrm{C}$ for 3 days and a dry weight (DW) determined. Moisture content was expressed as \% DW, as single determinations.

As a complementary study, samples from additional $E$. nitens saplings were used to determine moisture content as a proportion of saturated sample weight. Two trees were harvested, eight samples (approx. $2 \times 1 \times 1 \mathrm{~cm}$ ) of healthy sapwood were prepared and the fresh weight obtained immediately. For infiltration the samples were immersed in water until they sank and were then placed in a vacuum chamber for $5 \mathrm{~h}$. Samples were removed from the water, quickly surface dried by blotting and weighed to gain the saturated weight (SW). Samples were then dried at $85^{\circ} \mathrm{C}$ for 3 days and a dry weight determined. Moisture content was expressed as \% DW and $\%$ SW.

\section{Potassium concentration}

A single tree was wounded with a $6 \mathrm{~mm}$ drill bit at eight positions on the stem, creating wounds approx. $6 \mathrm{~mm}$ deep. Each wound was inoculated with a plug of G. adspersum agar culture and sealed with parafilm. Small drill wounds were used instead of notch wounds to reduce structural weakening of the stem. After 55 days the tree was harvested and three wounds were sampled. Plant material (60-150 mg FW) was excised from positions corresponding to the reaction zone (above and below, representing two "replicates") and from the opposite side of the stem, representing healthy sapwood. Fresh weight was determined and samples were placed in $5 \mathrm{ml} 1 \mathrm{M}$ nitric acid, heated to $80^{\circ} \mathrm{C}$ for $24 \mathrm{~h}$ and then allowed to digest for $2-3$ days. Plant material was then removed and the solution diluted 20-fold. The samples were analysed by flame photometry to measure $\mathrm{K}^{+}$. Readings were related to a standard curve of $\mathrm{KCl}(0-80 \mu \mathrm{M})$ by regression analysis.

\section{RESULTS}

Morphology, anatomy and histochemistry

Infection of inoculated wounds by $G$. adspersum progressed relatively slowly (Table 1 ). Within the first month, discolouration and decay proceeded in a triangular shape axially above and below the wound and did not extend transversely. Visible evidence of decay was not apparent in samples studied until 14 days after wounding and inoculation, however fungal hyphae were observable within vessels in samples studied after 7 days (Table 1). In samples studied after 14 days, hyphae were detectable in vessels and other axial cells. Hyphae appeared to colonize xylem cells which were not living (i.e. did not stain with NBT). G. adspersum was re-isolated as the dominant fungus from wood-chips of decayed tissue from the 21 and 30 day old wounds. Some contaminant fungi (apparently non-hymenomytaceous, e.g. Trichoderma spp.) and bacteria were also isolated from wood-chips. In some sections from wounds of different ages, clamp connections could be seen, confirming that it was basidiomycete hyphae.

Brown/red discolouration was first evidenced in the cambial region (Table 1). Discolouration of the outer xylem was evident for wounds analysed after 3 days, extending at the most to $3 \mathrm{~mm}$ from the edge of one wound. Discolouration around wounds tended to be orange/brown in colour at the interface of infection. Evidence of tylose formation (as unlignified material 
TABLE 1. Observation of infection and defence responses in wounded and inoculated $\mathrm{E}$. nitens

\begin{tabular}{|c|c|c|c|c|c|c|c|}
\hline & $12 \mathrm{~h}$ & $24 \mathrm{~h}$ & 3 days & 7 days & 14 days & 21 days & 30 days \\
\hline \multicolumn{8}{|l|}{ Infection } \\
\hline Decay (axial extent) & - & - & - & - & $1-2 \mathrm{~mm}$ & $2-3 \mathrm{~mm}$ & $1-5 \mathrm{~mm}$ \\
\hline Hyphae & - & - & - & $+\mathrm{V}$ & $+++\mathrm{VA}$ & $+++\mathrm{VA}$ & $++++\mathrm{VA}$ \\
\hline \multicolumn{8}{|l|}{ Responses } \\
\hline Cell death & + & + & + & ++ & +++ & +++ & ++++ \\
\hline Discolouration & C & $\mathrm{C}$ & $\mathrm{C}, \mathrm{OX}$ & $\mathrm{C}, \mathrm{X}$ & Pale & Pale & Varied \\
\hline PhenoLs & - & + & ++ & ++ & +++ & +++ & +++ \\
\hline Tyloses & - & $\operatorname{tr}$ & + & ++ & +++ & +++ & +++ \\
\hline Callus & - & - & - & + & + & +++ & ++++ \\
\hline Suberin & - & - & - & - & - & - & - \\
\hline
\end{tabular}

$\mathrm{C}=$ cambial, $\mathrm{OX}=$ outer xylem, $\mathrm{X}=$ xylem, $\mathrm{V}=$ vessels, $\mathrm{VA}=$ vessels and other axial cells, tr $=$ traces, + signifies extent of response.

within vessels) and phenol accumulation were first observed in samples studied $24 \mathrm{~h}$ after wounding and inoculation, becoming more extensive in older wounds. Phenol accumulation was observed both in association with vessel tyloses and in axial parenchyma. General accumulation of phenols was apparent with FBRRS up to $18 \mathrm{~mm}$ from the wound in samples studied after 14 days. Discolouration was less evident in wounds analysed between 14 and 30 days following wounding and inoculation, compared to the younger wounds. Although discolouration was pale, histochemical detection revealed a prominent accumulation of phenols at the interface of the infected wood (Fig. 1) with a general accumulation of phenols detected axial and adjacent to this, greater than on the opposite side of the stem. Callus tissue began to form by 7 days after wounding and inoculation.

Samples studied after 30 days showed some variation in colonization and response. That is, for both individual trees, one wound was considerably infected (approx. $5 \mathrm{~mm}$ axially) while the second wound on the same tree was infected much less (approx. 1-2 mm axially). At this stage an area of water-soaked appearance ahead of the decay was evident in some cases which was not observed in previous stages. Suberin was not detected by fluorescence microscopy in sections of the 30 day old wounds.

The single 72 day old wound (analysed by NMR imaging) had almost completely callused over and a barrier zone and new xylem were also present. Decay spread axially from the wound to $3-4 \mathrm{~mm}$ and also transversely to $1-2 \mathrm{~mm}$. A prominent reaction zone had formed axially, including both orange and brown zones.

\section{Analysis of phenolic compounds}

The levels of total phenols in the healthy sapwood samples remained essentially constant while the levels in the wood surrounding the wound (RZ) increased linearly to a peak at 21 days (Fig. 2). This peak represents a four- fold increase in phenolics on average. After 21 days the total phenol levels began to decrease. HPLC analysis of the methanol extracts revealed that qualitative changes in phenols occurred within the time-scale of the experiment (Fig. 3). Major peaks in the chromatograms have been labelled alphabetically. Comparison of chromatograms shows that many more compounds were detected in the developing reaction zone compared to healthy sapwood. This includes peaks $\mathrm{D}$ and $\mathrm{E}$ and a range of other peaks

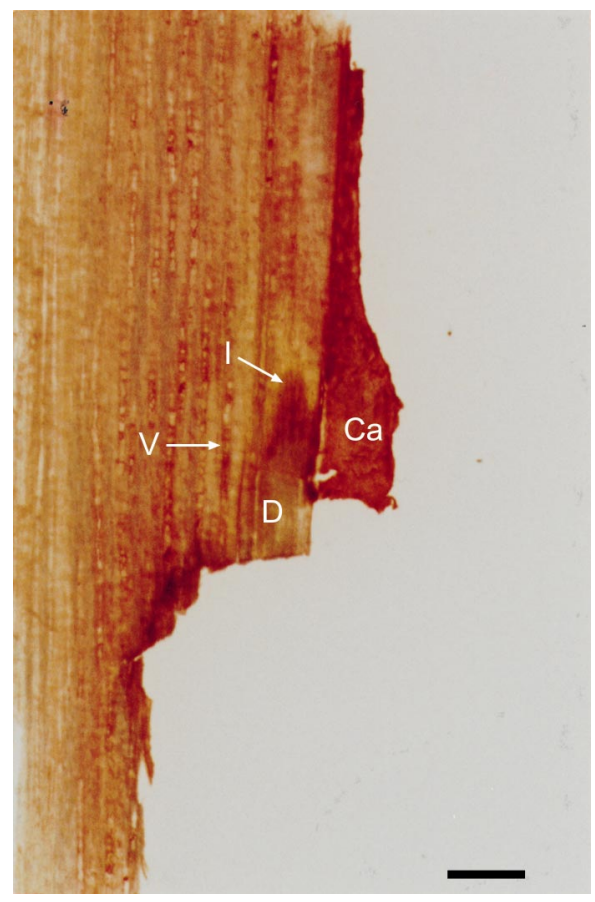

FIG. 1. Radial longitudinal section below a 21 day old inoculated wound, stained with FBRRS. A discrete region of phenols is detectable at the interface (I) of decay column (D) and un-infected wood. General accumulation of phenolics surrounds this area, particularly associated with vessel tyloses, which can be visualized at this relatively low magnification. Callus tissue $(\mathrm{Ca})$ near the wound stains strongly for phenolics. Scale bar $=2 \mathrm{~mm}$. 


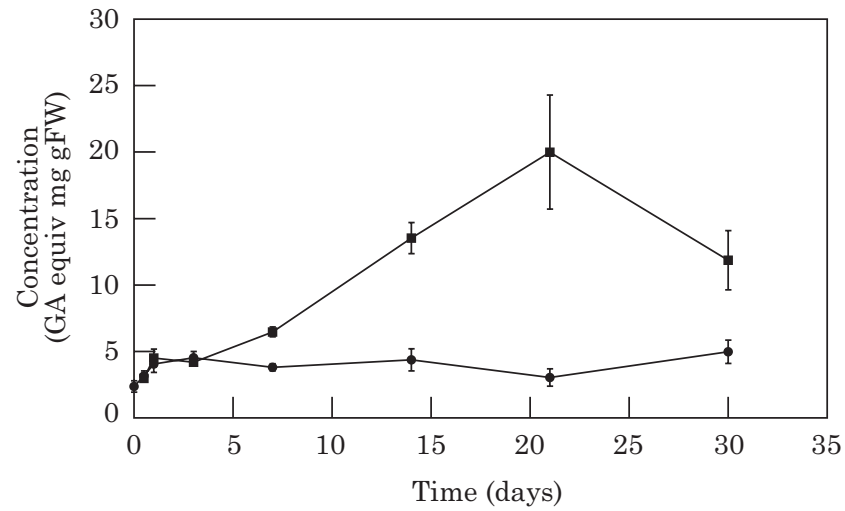

Fig. 2. Total phenol levels (mg GA equivalent $\mathrm{gFW}^{-1} \pm \mathrm{SE}$ ) determined from methanol extracts (four replicates from two tress) of reaction zone $(\mathbf{\square})$ and healthy sapwood $(\boldsymbol{\bullet})$ over time following wounding and inoculation.

which cannot be fully distinguished due to relatively poor separation.

With the use of an external gallic acid standard, semiquantification was acquired for selected prominent peaks (Fig. 4). Peak Q showed a dramatic increase in concentration $(3 \cdot 5$-fold $)$ within the first $24 \mathrm{~h}$ after wounding and challenge and then remained at a similar level for the experimental period. Peaks D and E increased steadily until 21 days, following the trend found for the total phenol levels (Fig. 4). Comparison of retention times to samples subsequently studied with liquid chromatography-mass spectrometry (LC-MS) allows extrapolation of the nature of these compounds [2]. Peak $Q$ is a tetra-galloylglucose isomer, while peak $\mathrm{D}$ and $\mathrm{E}$ relate to two anomeric peaks of pedunculagin and their concentrations have therefore been combined (Fig. 4). The changes in concentration of peak D and $\mathrm{E}$ (Fig. 4, plotted separately) are similar, supporting the conclusion that they are related peaks.

\section{Potassium concentration}

The determinations of $\mathrm{K}^{+}$concentration for three 55 day old wounds from one tree are shown in Table 2. Apart from wound $1, \mathrm{~K}^{+}$was lower in the reaction zone compared to the healthy sapwood. On average the level of $\mathrm{K}^{+}$is lower in the reaction zone, although this is not statistically significant $(t$-test, $P>0 \cdot 2)$.

\section{Moisture content}

Images of the first echo, $M_{0}$ map and $T_{2}$ map were obtained for each tree in the time-course experiment. Due to background noise in the $M_{0}$ and $T_{2}$ maps, the first echo maps are presented of the sequence of NMR images from wounds of different ages [Fig. $5(\mathrm{a})-(\mathrm{h})]$. Because of the uniformity of $T_{2}$ values, the contrast in the first echo
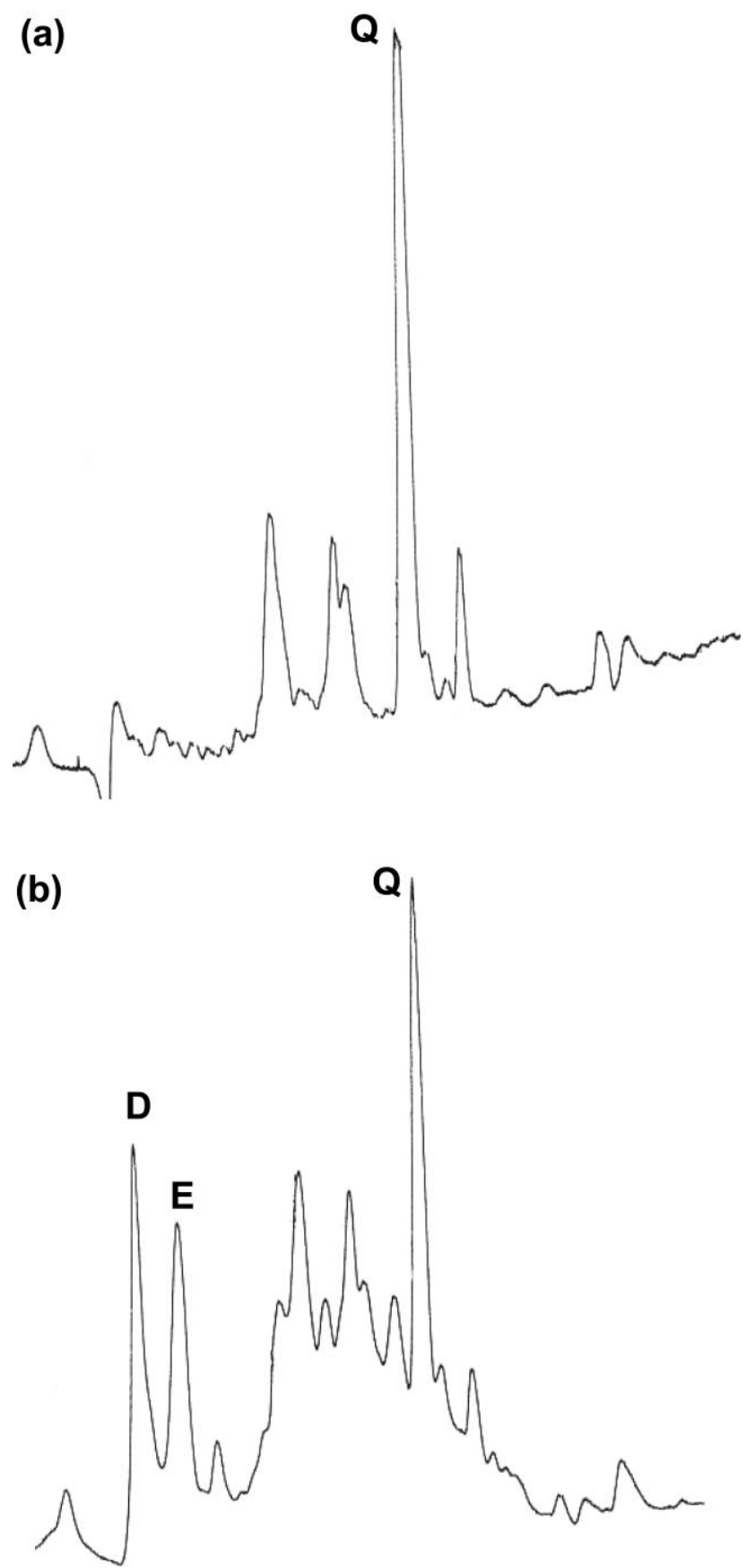

Fig. 3. HPLC chromatograms (detected at $260 \mathrm{~nm}$ ) of methanol extracts from E. nitens; (a) unwounded wood and (b) reaction zone region 30 days after wounding.

and $M_{0}$ map was virtually identical. $M_{0}$ values cannot be cross-referenced from tree to tree, but are comparable within the same tree.

Image of the control tree [Fig. $5(\mathrm{a})]$ indicates that the outer xylem tissue is highest in moisture content, compared to the older xylem and central pith. The bark is barely detectable and therefore reasonably dry. Axial striations in the xylem probably relate to the 


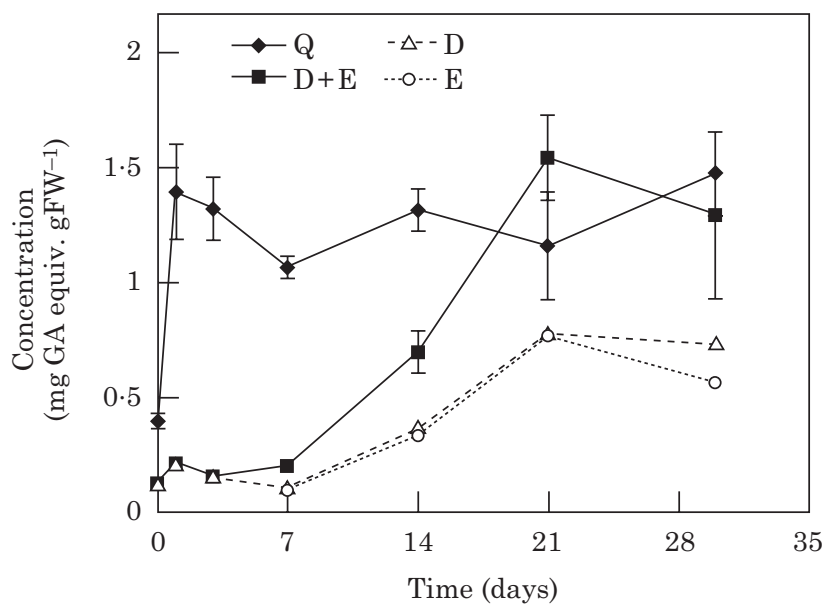

Fig. 4. Concentration of individual HPLC peaks (mg GA equivalent $\mathrm{gFW}^{-1} \pm \mathrm{SE}$ ) over time following wounding and inoculation.

TABLE 2. Concentration of $K^{+}\left(\mu M m g F W^{-1} \pm S E\right)$ in healthy sapwood and reaction zone samples determined from three wounds on $1 \mathrm{E}$. nitens tree (two replicates per tree)

\begin{tabular}{lcc}
\hline Wound & Healthy sapwood & Reaction zone \\
\hline 1 & $7 \cdot 91 \pm 0 \cdot 09$ & $8 \cdot 09 \pm 0 \cdot 34$ \\
2 & $7 \cdot 38 \pm 0 \cdot 87$ & $5 \cdot 74 \pm 0 \cdot 16$ \\
3 & $7 \cdot 28 \pm 0.54$ & $5 \cdot 51 \pm 0 \cdot 39$ \\
Average & $7 \cdot 52 \pm 0 \cdot 43$ & $6 \cdot 45 \pm 0.53$ \\
\hline
\end{tabular}

distribution of vessels and axial parenchyma. The gravimetric and $M_{0}$ results from this tree show considerable internal variability that does not correspond exactly between the two methods. This is probably a function of the difference in total volume measured by each method, as $M_{0}$ values were calculated from an area of 40 pixels from a $2 \mathrm{~mm}$ slice, while blocks excised for gravimetric studies were considerably bigger than this.

The results for the tree imaged within $1 \mathrm{~h}$ of wounding and inoculation showed that the contrast on the wounded side of the stem was lower, observable as a general area [Fig. 5(b)]. Gravimetric measurements also showed decreased moisture content on the wounded side of the stem [Fig. 5(b)]. $M_{0}$ results for the stem analysed $24 \mathrm{~h}$ after wounding [Fig. 5(c)] indicate that moisture content is unchanged, although gravimetric results indicate a drop in moisture below the wound. Results for the stem analysed 4 days after wounding [Fig. 5(d)] are confounding, with little change in moisture content determined gravimetrically but decreases and increases in $M_{0}$ above and below the wound, respectively.

Analysis of a stem 7 days after wounding revealed a distinct dry zone observable above and below the inoculated wound. Although $M_{0}$ values could not be calculated for this tree because of the low signal to noise ratio, a substantial drop in moisture content around the wound of normal xylem levels was indicated by the gravimetric results. This drying was not as prominent around the wounds at later stages; the tree imaged with a 15 day old wound [Fig. 5(f)] showed a decrease in $M_{0}$ above and below the wound, which was evidenced to a lesser extent by gravimetric data.

Around the 22 day old wound [Fig. 5(g)] some evidence of moisture content increase is apparent from both the $M_{0}$ values and gravimetric data, particularly below. Areas of bright contrast are observable above and below the wound after 72 days [Fig. $5(\mathrm{~h})]$. These areas correspond with the reaction zone and $M_{0}$ values are greater than the adjacent healthy sapwood. Gravimetric determinations of moisture content did not detect these increases. The progress of decay around the wound is evidenced by a dark region in the images, with low $M_{0}$ and gravimetric values adjacent to the wound.

Complementary studies of $E$. nitens xylem moisture content as a percentage of saturated weight revealed an average of $55.35 \% \mathrm{SW}( \pm 0.58 \mathrm{SE})$ which related to an average of $179 \cdot 76 \% \mathrm{DW}( \pm 3 \cdot 16 \mathrm{SE})$.

\section{DISCUSSION}

$G$. adspersum colonized E. nitens stem wounds to a limited extent and therefore represented a non-aggressive fungalplant interaction. Responses to wounding and inoculation included parenchyma cell death, phenolic production beginning within $24 \mathrm{~h}$, tyloses formation and slight alterations in potassium levels and water content. Upon wounding, the withdrawal of water from severed xylem cells and ingress of oxygen may instigate a number of these responses $[23,29]$. The extent of colonization may then determine the degree of development of these responses. That is, the fact that the inoculated E. nitens 21 and 30 day old wounds showed considerable variation in colonization and discolouration (Table 1) and phenolics content (Figs 2 and 4) indicates that xylem responses to wounding may be secondary to responses to fungal colonization. Why some wounds became more colonized than others was difficult to determine.

The rapid accumulation of compounds detected by total phenol assays and HPLC suggest that phenolics are particularly important in E. nitens sapwood defence (Figs 2 and 4). Hydrolysable tannins may be effective to halt microbial growth by binding proteins such as fungal enzymes $[9,15]$. Alternatively the ability of tannins to bind metals [19] or act as antioxidants [12] may restrict fungal metabolism. Tetra-galloylglucose may be a key precursor for ellagitannin formation [11, 13], which would explain the quick increase in tetragalloylglucose levels, followed by slower increases in pedunculagin levels (Fig. 4). 

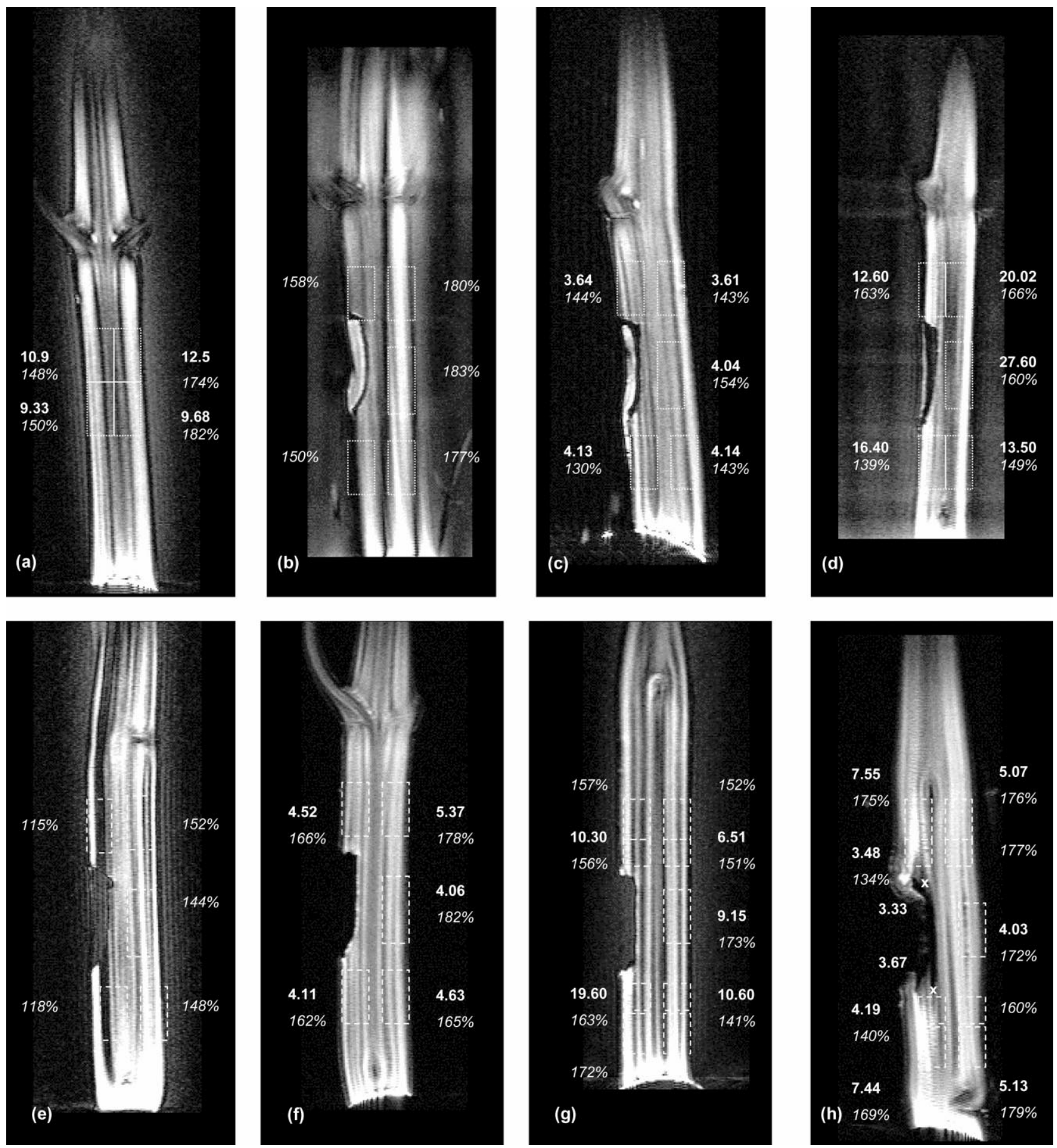

FIG. 5. NMR images (first echo) for each intact pot-grown E.nitens sapling, control (a) and various stages following wounding and inoculation with $G$. adspersum, within $1 \mathrm{~h}$ (b), $24 \mathrm{~h} \mathrm{(c)} \mathrm{and} 4$ days (d). Boxes super-imposed on maps (dashed lines) represent approximate position of wood samples excised for gravimetric determination of moisture content, with results expressed in italics (single determinations). $M_{0}$ values (calculated at the centre of these positions) are expressed in bold. First echo maps of $E$. nitens saplings at various stages after wounding and inoculation; 7 days (e), 15 days (f), 22 days (g) and 72 days (h). Additional points where $M_{0}$ values were determined are marked with x.

NMR imaging has allowed a non-invasive study of xylem moisture content in wounded and inoculated E. nitens. In the interpretation of these images a number of factors must be considered, including the effects of magnetic susceptibility [26]. That is, samples with a high moisture content (such as E. nitens) will result in an increase of image contrast at water-oxygen interfaces. Results obtained present a number of differences 
compared to a time-course experiment completed for A. pseudoplatanus [24] in which drying of xylem was apparent in the first 2 days, followed by re-wetting between the period 5 and 39 days. In E. nitens there was NMR imaging evidence for substantial drying 7 days after wounding [Fig. 5(e)]. This was supported by gravimetric analysis, which indicated that the decrease in moisture was to $76 \%$ of normal xylem levels. It could be postulated that due to the high moisture content of the E. nitens saplings, a dry level critical for colonization takes longer to result than other hardwood trees such as sycamore. Although E. nitens may have a high moisture content when based on dry weight, determinations based on saturated weight reveal that even at a moisture content of $170 \% \mathrm{DW}$ the wood is far from saturated. That is, the experiments involving water-infiltrated healthy sapwood samples revealed that typical moisture contents were $55 \%$ when based on saturated weight.

Although drying does not seem to be dramatic, the withdrawal of water from vessels following wounding must be sufficient to instigate the accumulation of phenols and formation of tyloses (however, elicitation processes by fungi may also prove to induce these factors). Phenol biosynthesis appears to require the presence of oxygen $\{[8,29]$ R.B. Pearce, unpublished work $\}$ and the accumulation of phenols can be detected in E. nitens from $24 \mathrm{~h}$ after wounding. The formation of tyloses is also observable shortly after wounding, with evidence for formation within $24 \mathrm{~h}$ in E. nitens (Table 1). Necesany [21] found that tyloses formation in Eucalyptus botryoides sapwood increased with exposure to air. In E. nitens it was found that most tyloses development had occurred by 14 days (Table 1). Tyloses may be effective to block the vessels and prevent any further drying, allowing a return to equilibrium by apoplastic diffusion, as evidenced by $M_{0}$ and gravimetric data for the 15 day wound. In the discussion of these results, it is important to consider that individual trees were imaged in this experiment, and the progress of infection and defence responses may have varied in different trees at different stages.

Water soaking was evident in advance of infection in E. nitens by at least 30 days. NMR imaging detected an accumulation of water in the trees with inoculated wounds aged 22, 42 and 72 days, but this was not represented by results from gravimetric measurements. This suggests the increase was not great enough to be detected by gravimetric methods, in contrast to sycamore. $M_{0}$ values from the 72 day old wound [Fig. 5(h)] showed that this accumulation is approx. 1.5 times that of the healthy sapwood on the opposite side of the stem. As mentioned, magnetic susceptibility may accentuate this accumulation. Pearce [26] noted that the $M_{0}$ value in A. pseudoplatanus infected with Ustilina deusta or G. adspersum (both non-aggressive fungi) was between 2.9 and 3.7 times higher, while the increase measured gravimetrically was only $1 \cdot 1-1.7$ times. As the difference between $M_{0}$ values in E. nitens is not to this extent it can be concluded that water accumulation is not as prominent as in A. pseudoplatanus. A conservative evaluation of the data suggests that moisture content returns to typical or slightly higher levels in the compromised E. nitens xylem.

It appears that a different system of response occurs in E. nitens compared to A. pseudoplatanus. Firstly, large increases in potassium and calcium ions occur in the A. pseudoplatanus reaction zone [10] which may osmotically drive the accumulation of water to the site. As a general decrease in potassium ions has been evidenced in the E. nitens reaction zone $\left\{\right.$ and decreases in $\mathrm{K}^{+}, \mathrm{Ca}^{2+}$, $\mathrm{Mn}^{2+}$ and $\mathrm{Mg}^{2+}$ in previous studies [3] $\}$ this could not account for the moisture accumulation detected. Other means of increasing moisture content include increases of solubilized sugars or sugar alcohols in the osmoticum. This could be related to the accumulation of hydrolysable tannins (glucose based phenols) in the reaction zone. More likely, the production of tyloses and polyphenolic deposits reduce water flow resulting in a physiological "backwater" where water accumulates. This is more easily related to accumulation below the wound than above as bulk water flow in the xylem occurs axially through vessels, however diffusion also occurs through living cells such as ray parenchyma $[38]$ which may direct water above the wound.

In conclusion, this study has outlined the induction of key defence responses in E. nitens xylem in the early stages following wounding and inoculation. As opposed to the model for angiosperms proposed by Pearce [23], E. nitens does not accumulate moisture within the first week following wounding. Moisture accumulation was evidenced in the reaction zone by NMR imaging, but may be a result of the physiological changes associated with wounding and infection rather than as an active defence as it was not observed until later in the interaction. However, rapid accumulation of phenolics and formation of tyloses does occur in E. nitens and may be the most important features of defence.

K. M. Barry is gratefully funded by an Australian Postgraduate Award with industry support from the Tasmanian Forest Research Council. This research was completed during a research trip to the U.K. by K. M. Barry, made possible by a British Council Postgraduate Bursary. Thanks to E. Peacock (University of Tasmania, Hobart) and K. T. Smith (USDA Forest Service) for helpful discussions. Revision of the manuscript by G. Kile and G. Downes (CSIRO Forestry and Forest Products) is appreciated. The experience of Ray Pearce was an immense contribution to this project and he is sadly missed. 


\section{REFERENCES}

1. Asiegbu FO, Johansson M, Woodward S, Hüttermann A. 1998. Biochemistry of the host-parasite interaction. In: Woodward S, Stenlid J, Karjalainen R, Hüttermann A, eds. Heterobasidion Annosum: Biology, Ecology, Impact and Control. CAB International.

2. Barry KM, Davies NW, Mohammed GL. Identification of hydrolysable tannins in the reaction zone of Eucalyptus nitens wood, by high performance liquid chromatography/ electrospray ionization mass spectrometry. Phytochemical Analysis. In press.

3. Barry KM, Pearce RB, Mohammed GL. 2000. Properties of reaction zones associated with decay from pruning wounds in plantation-grown Eucalyptus nitens. Forest Pathology 30: 233-245.

4. Boddy L, Rayner ADM. 1983. Origins of decay in living deciduous trees: the role of moisture content and a reappraisal of the expanded concept of tree decay. New Phytologist 94: 623-641.

5. Bonello P, Pearce RB. 1993. Biochemical defence responses in primary roots of Scots pine in vitro with Cylindrocarpon destructans. Plant Pathology 42: 203-211.

6. Brown JM, Johnson GA, Kramer PJ. 1986. In vivo magnetic resonance microscopy of changing water content in Pelargonium hortorum roots. Plant Physiology 82: 1158-1160.

7. Connelly A, Lohman JAB, Loughman BC, Quiquampoix H, Ratcliffe RG. 1987. High resolution imaging of plant tissues by NMR. Fournal of Experimental Botany 38: 1713-1723.

8. Coutts MP. 1977. The formation of dry zones in the sapwood of conifers. II. The role of living cells in the release of water. European Fournal of Forest Pathology 7: 6-12.

9. Field JA, Lettinga G. 1992. Toxicity of tannic compounds to microorganisms. In: Hemingway RW, Laks PE, eds. Plant Polyphenols. New York, U.S.A.: Plenum Press.

10. Grime GW, Pearce RB. 1995. External beam analysis of living sycamore xylem infected by pathogenic fungi. Nuclear Instruments and Methods in Physics Research B 104: 299-305.

11. Gross GG. 1992. Enzymes in the biosynthesis of hydrolysable tannins. In: Hemingway RW, Laks PE, eds. Plant Polyphenols. New York, U.S.A.: Plenum Press, 43-60.

12. Hagerman AE, Riedl KM, Jones A, Sovik KN, Ritchard NT, Hartzfeld PW, Riechel TL. 1998. High molecular weight plant polyphenolics (tannins) as biological antioxidants. F. Agric. Food Chem. 46: 1887-1892.

13. Helm RF, Zhentian L, Ranatunga T, Jervis J, Elder T. 2000. Towards understanding monomeric ellagitannin biosynthesis. In: Gross G, Hemingway RW, Yoshida T, eds. Plant Polyphenols 2: Chemistry and Biology. New York, U.S.A.: Plenum Press, 83-99.

14. Johansson M, Stenlid J. 1985. Infection of roots of Norway spruce (Picea abies) by Heterobasidion annosum I. Initial reactions in sapwood by wounding and infection. European Fournal of Forest Pathology 15: 32-45.

15. Kawamoto H, Mizutani K, Nakatsubo F. 1997. Binding nature and denaturation of protein during interaction with galloylglucose. Phytochemistry 46: 473-478.

16. Lohman JAB, Ratcliffe RG. 1988. Prospects for NMR imaging in the study of biological morphogenesis. Experentia 44: 666-672.

17. Marquardt DW. 1963. An algorithm for least-square estimation of non linear parameters. Fournal of Society and Industrial Applied Mathematics 11: 431-441.
18. Meiboom S, Gill M. 1958. Modified spin-echo method for measuring nuclear relaxation times. Review of Scientific Instruments 29: 688-691.

19. Mila I, Scalbert A, Expert D. 1996. Iron withholding by plant pathogens and resistance to pathogens and rots. Phytochemistry 42: 1551-1555.

20. Murmanis L. 1975. Formation of tyloses in felled Quercus rubra L. Wood Science and Technology 9: 3-14.

21. Necesany V. 1973. Kinetics of secondary changes in living xylem. I. Time dependent formation of tyloses and polyphenolic substances. II. Some biophysical aspects. Holzforschung 27: 73-79.

22. Pearce RB. 1990. Occurrence of decay-associated xylem suberization in a range of woody species. European fournal of Forest Pathology 20: 275-289.

23. Pearce RB. 1996. Antimicrobial defences in the wood of living trees. New Phytologist 132: 203-233.

24. Pearce RB. 1998. An integrated model for antifungal defence in the functional xylem (sapwood) of Acer pseudoplatanus (Sycamore). Abstracts of the 7 th International Congress of Plant Pathology, August 9-16, Edinburgh, U.K. Abstract no. 1.9.25.

25. Pearce RB. 2000. Decay development and its restriction in trees. Fournal of Arboriculture 26: 1-11.

26. Pearce RB, Fisher BJ, Garpenter TA, Hall LD. 1997. Water distribution in fungal lesions in the wood of sycamore, Acer pseudoplatanus, determined gravimetrically and using nuclear magnetic resonance imaging. New Phytologist 135: 675-688.

27. Pearce RB, Sümer S, Doran SJ, Carpenter TA, Hall LD. 1994. Non-invasive imaging of fungal colonization and host response in the living sapwood of sycamore (Acer pseudoplatanus L.) using nuclear magnetic resonance. Physiological and Molecular Plant Pathology 45: 359-384.

28. Pearce RB, Woodward S. 1986. Compartmentalization and reaction zone barriers at the margin of decayed sapwood in Acer saccharinum L. Physiological and Molecular Plant Pathology 29: 197-216.

29. Rayner ADM, Boddy L. 1988. Fungal Decomposition of Wood. Chichester, U.K.: John Wiley \& Sons.

30. Shain L. 1967. Resistance of sapwood in stems of loblolly pine to infection by Fomes annosus. Phytopathology 57: 1034-1045.

31. Shain L. 1971. The response of sapwood of Norway Spruce to infection by Fomes annosus. Phytopathology 61: 301-307.

32. Shain L. 1979. Dynamic responses of differentiated sapwood to injury and infection. Phytopathology 69: 1143-1147.

33. Shigo AL. 1967. Succession of organisms in discolouration and decay of wood. International Review of Forestry Research 2: $237-299$

34. Shigo AL. 1972. Succession of microorganisms and patterns of discolouration and decay after wounding in red oak and white oak. Phytopathology 62: 256-259.

35. Shortle WG, Smith KT. 1990. Decay column boundary layer formation in maple. Biodeterioration Research 3: 377-389.

36. Van As H, Schaafssma TJ. 1984. Non-invasive measurement of plant water flow by nuclear magnetic resonance. Biophysical fournal 45: 469-472.

37. Yamada T. 1992. Biochemistry of gymnosperm xylem responses to fungal invasion. In: Blanchette RA, Biggs AR, eds. Defence Mechanisms of Woody Plants Against Fungi. Berlin: Springer-Verlag, 147-164.

38. Zimmerman MH. 1983. Xylem Structure and the Ascent of Sap. Berlin: Springer-Verlag. 